\title{
CORRESPONDENCE.
}

\section{IS ELECTRICITY FORCE OR MATTER?}

\section{Editors of The Franklin Institute Journal:}

Dear Sirs:-During the past few years, I have advanced the following argument to a number of electricians, and, having failed to find anyone who can adduce any good argument against it, I thought it might be of sufficient interest to be discussed in your journal. Possibly it may not be new, but $I$ have never seen it published elsewhere.

- I believe it is conceded that everything in the universe is either force or matter; therefore, electricity must be one or the other. If it is matter, it must remain the same in amount, and can never be consumed or generated. If it is force, it may be generated by the expenditure of another force-as that in the energy of a steam engine-and will then grow less in amount as it is again converted into other forces-as in the energy of motors, lamps, or in heating wires. Now, it is a well-known fact that quantity of electricity measured in coulombs, never is generated, never is consumed, and never does grow less in the circuit, barring leakage. The current flowing out of a lamp is exactly the same in quantity as that flowing into it, the same being true of motors and of generators, showing that electricity itself is neither consumed while doing work, nor is it generated; after doing work in a lamp or motor, it comes out in precisely the same quantity as it entered. Connect only one pole of a hattery to a circuit and there will be no current. Why? The battery is not able to generate quantity, or coulombs, of electricity; all it is able to do is to take the quantity which flows in at the negative pole and to send it out at the positive pole, with an increased pressure or electromotive force. The battery, therefore, does not generate electricity, but merely raisesthe pressure of that which flows in. Electricity, therefore, appears to be matter, but not force. It is precisely analogous to water in a water circuit. The water is neither consumed nor generated. The pump merely increases the pressure of the water which flows in at one end; the water motor or turbine consumes this pressure again, converting it into mechanical work of another kind; $\mathbb{t}$ does not consume the water. The quantity of water measured in units of quantity, is the same in all parts of the same closed circuit of water, just as the quantity of electricity in an electric circuit. The work which an electric current can do is due to the pressure or electro-motive force; without pressure it can do no work. The electricity in the earth is like the water in the ocean-neither can do work unless raised to some pressure or height, or allowed to fall below its normal level.

The term force is used here as distinguished from energy, as the latter term might be construed to include in it the conception of matter. But even if matter and energy constitute the universe, the above argument applies equally well. It is understood, of course, that by the term electricity as measured in coulombs, is not meant electrical energy as expressed in watts or joules, for it is beyond question that the latter is energy. 
Perhaps it will be found, at some future time, as has been already suggested, that electricity is the ether (which is believed to be matter) whose wave motions are light, and which, in some other form of motion, is an electric current. Perhaps a current of electricity is the bodily conveyance of ether, as distinguished from a wave motion, which is energy in the form of light. In that case, I would suggest that perhaps the relative motion of the ether of space, and the revolving earth may explain the cause of the earth's magnetism, the ether in motion around the earth (relatively) being an electric current producing magnetic effects. The magnetic polarity and direction at the equator are in accordance with such a theory, and the fact that the lines of force bend down into the earth at the magnetic poles may be explained by the wellknown fact that lines of force are continuous circuits; they must return somewhere, and they select the axis of the earth, as there is no motion there to develop a counter-magnetism. They cannot return outside of the earth, as lines of force cannot intersect each other, and, in order to return outside of the earth, they would have to intersect. Furthermore, observations show that they do not return outside of the earth.

The only plausible arguments which I can find against the theory that electricity is matter, are that it may be a combination of force and matter, as, for instance, a wave motion; or, it may be, that the real current is in the same direction in both wires leading from a machine, therefore emanating from the machine, and, consequently, being force or energy.

Yours very truly,

Carl Hering.

Philadelphia, April 18, 1887 .

\section{SCIENTIFIC NOTES AND COMMENTS.}

\section{ASTRONOMY AND PHYSICS.}

The Observatory of Meudon.-M. Janssen (Comptes Rendus, 104, r,067), as President of the Academy of Sciences at its meeting, April i 8 th, gave cordial welcome to the members of the International Conference of celestial photography, and in so doing invited attention to the observatory at Meudon. Regarded as a "vote of confidence" for the new astronomy, the extranrdinary equipments now being put up at this observatory are in the highest degree expressive. As is well known, the instruments for the photographic study of the sun here long in use, have been specially adapted to the work, and by the ingenious methods of $M$. Janssen, have added not a little to our knowledge.

But now colossal additions are to be made. A reflector of I metre in diameter and of only about 3 metres focal length, and consequently giving abundant light to images of sensible size, is provided for the optical, spectroscopic or photographic study of faint nebulæ, comets, etc. The mirror, already completed by the MM. Henry, is considered of rare perfection.

As equatorial, the observatory is to have a huge twin instrument, one refractor of $\mathrm{o}^{\mathrm{m}} \cdot 8 \mathrm{I}$ ( $32 \mathrm{in}$.) aperture, to be devoted to spectroscopy and the ordinary Whole No. Vol. CXXIII.-(Third Series, Vol. xciii.) 